\title{
Visualizing the Organization of Mitotic Chromosomes by Super-resolution Microscopy
}

\section{Hu Cang}

\author{
Salk Institute for Biological Studies, San Diego, California, United States
}

Mitosis is one of the most dramatic events of cells. In less than 40 minutes chromatin condense and fold into X-shaped chromosomes; folding errors could contribute to chronotherapies, aneuploidy, chromosome translocations, and infertility; yet the mechanisms underlying the folding remain largely unknown, hindering the progress in finding cures to mitotic errors.

Chromosome scaffold, a proteinous structure, comprising mainly topoisomerase $2 \mathrm{a}$ (top $2 \mathrm{a}$ ) and condensin in metaphase, spanning the full length at the core of each chromatid, is the machinery that is responsible for chromosome folding. The scaffold appears in early pro-phase and disassembles in telophase. Knocking down either top2a or condensin is sufficient to disrupt chromosome assembly, and co-incubation of the two with nucleosome-free DNA could reconstruct chromatid-like structures in vitro, suggesting that chromosome scaffold holds the key to the understanding of chromosome folding, and its misfunctions could cause mitotic errors.

However, the structure of chromosome scaffold remains largely unknown, reflecting the need for a microscopy technique with higher resolution. Electron microscopy, while has sufficient resolution, lacks specificity to pinpoint individual molecules. Therefore, chromosome scaffold was discovered after depleting histones from a chromosome. The histone-depletion step, which is necessary to expose the scaffold for electron microscopy, causes massive distortions. Indeed, both a fibrous architecture resembling an interconnected web of proteinous filaments and a compact and rod-like scaffold with a solid core has been observed. It is not clear which, or either, reflects the native architecture of chromosome scaffold.

In addition, whether top2a belongs to the scaffold or merely co-precipitate with condensin due to the harsh treatments remains unknown. Because top2a is a highly active enzyme that decatenates entangled DNA, top2a is not considered by most models as an integrated component of chromosome scaffold.

The invention of super-resolution microscopy including Palm/STORM, SIM and STED transformed optical microscopy. However, the most commonly achievable resolution suitable for chromosome studies, which is limited by factors such as sample drift, photobleaching, overlapping blinking, and optical aberrations is usually limited to about $\sim 30 \mathrm{~nm}$ in a thick sample. Recently, Chen et al. demonstrated Expansion Microscopy (ExM), which uses a hydrogel to mechanically magnifies the features of a specimen. The techniques undergo rapid developments to achieve homogeneous expansion at the molecular scale.

Here, we develop a platform integrating Palm/STORM with expansion microscopy. Expansion STORM enables visualizing chromosome scaffold directly and reveals that chromosome scaffold has a fibrous architecture. Unexpectedly, both top2a and condensin form filaments, which interlace together into the scaffold. Furthermore, expansion STORM finds that the fibrous scaffold is established in two steps. First, top2a and condensin filaments braid together into a single- axis scaffold, which then splits into two, one for each chromatid, giving rise to the iconic X-shape. Lastly, the braid-split process requires cooperation between top2a and condensin, mediated by the $\mathrm{C}$-terminal domain of top2a. Antagonizing the cooperation 
disrupts chromosome assembly, blocks chromatid resolution, and leads to spherical, instead of rod-shaped chromatids. Although top2a and condensin form filaments in early prophase, because the filaments are thin and sparse, they are difficult to detect by other means. Therefore, expansion STORM demonstrates its unique capabilities for interrogating chromosome organizations.

\section{References}

$\mathrm{Xu}$, Huizhong, et al. "Molecular organization of mammalian meiotic chromosome axis revealed by expansion STORM microscopy." Proceedings of the National Academy of Sciences 116.37 (2019): 1842318428. 